\title{
UNA APROXIMACIÓN EMPÍRICA AL CAPITAL HUMANO MUNICIPAL EN CHILE
}

\author{
HÉctor Ormeño Campos (hector.ormenoc@gmail.com) \\ Investigador Asociado \\ Instituto Chileno de Estudios Municipales \\ Universidad Autónoma de Chile
}

En este artículo se entrega evidencia con respecto a la productividad de los funcionarios municipales. A través de la construcción de una base de datos con información del Sistema Nacional de Información Municipal, grados de salario del alcalde y un índice de producto municipal, se comprueba la hipótesis de un efecto positivo de los funcionarios sobre el producto municipal. Los hallazgos permiten comprobar la hipótesis de rendimientos marginales decrecientes de los funcionarios sobre el producto municipal y un efecto positivo de los profesionales sobre el producto.

Palabras clave: Capital humano; producto municipal; productividad; funcionarios 


\section{AN EMPIRICAL APPROACH TO MUNICIPAL HUMAN CAPITAL IN CHILE}

This paper provides evidence with respect to the productivity of municipal workers. The hypothesis that municipal workers have a positive effect on municipal product is based on a database from the National System of Municipal Information (SINIM), mayors' salary scales and an index of municipal product. The findings confirm the hypothesis of decreasing marginal returns, while professional staffs have a positive effect on the total municipal product.

Keywords: Human capital; municipal product; productivity; public employees 
El concepto de capital humano ha cobrado especial importancia en los últimos tiempos en Chile. Conocidas han sido las iniciativas que se han llevado a cabo con el objeto de aumentar la escolaridad de la población, tal como ocurrió con la reforma constitucional que establece la obligatoriedad de la educación secundaria el año 2003. Este mayor nivel de educación genera un mayor retorno para las personas, una mayor productividad laboral y desde luego, ciudadanos más informados y conscientes de sus deberes y derechos, todas características fundamentales para el mejor funcionamiento de la democracia. La desigualdad del ingreso también se ve influenciada por la educación (Meller, 2000; Contreras, 1999; Beyer, 1997), y corresponde a un factor que influye la convivencia social, el bienestar, y el mayor logro de consensos.

Este estudio se sitúa en un contexto más específico y particular del estudio capital humano, que tiene que ver con el sector público, y particularmente con las municipalidades. En este panorama, la razón que nos lleva a estudiar el capital humano de las municipalidades esta implícitamente relacionada al rol que este organismo tiene en el territorio, y por ende, sobre el bienestar de las comunidades locales.

La productividad del funcionario municipal ha permanecido en las sombras, debido al problema de medir el producto municipal, como a la poca disponibilidad de datos sobre las características de los funcionarios del sector. Este fenómeno es consecuencia del bajo interés por las temáticas locales, desplazadas por el excesivo centralismo del país, el que se refleja en la opinión pública, en los medios, y en la forma de diseñar la política pública.

Este estudio, en la línea de Zamorano, González y Lagos (2010), es un intento por develar algunos hechos básicos en el capital humano municipal. En particular, en lo referente a la productividad de los funcionarios, lo que interesa es observar el efecto sobre el producto municipal de ciertas variables asociadas al capital humano, tales como el número de funcionarios per cápita, el nivel de profesionalización, y la experiencia, entre otras.

De acuerdo a lo anterior, el aporte de este artículo es presentar un modelo basado en datos empíricos, que da cuenta de la productividad de los funcionarios municipales, y que usa para ello una medida de producto municipal que pretende considerar la amplitud de la gestión municipal. 
Este trabajo cuenta con seis secciones. En la primera se realiza una breve descripción de la literatura, y en la segunda se discute con respecto a una potencial medida de producto municipal. En la tercera se discute sobre los resultados esperados. En la cuarta sección se describen los datos a usar en el ejercicio, y en la quinta se exponen los resultados. Por último, en la sexta sección se exponen las conclusiones de este estudio.

\section{LITERATURA SOBRE CAPITAL HUMANO: HECHOS ESTILIZADOS}

Adam Smith destacó el papel de la educación, el aprendizaje a través de la experiencia, y la formación técnica en los determinantes de las posibilidades de producción (Sen, 1997: 70). Este aporte seminal ha sido la base de posteriores desarrollos que imputan empíricamente la mayor productividad de las personas en el proceso de producción de bienes y servicios a factores relacionados directamente a la educación y la experiencia. El primero se vislumbra como un tipo de inversión que las personas llevan a cabo en sí mismas, y el segundo surge de forma inherente durante el proceso de producción. Conocida es la famosa frase en la cual Smith (1776) compara un hombre educado a expensas de trabajo y tiempo, a una costosa máquina.

Dentro de las líneas más tradicionales del estudio del capital humano destacan dos enfoques, uno microeconómico y otro macroeconómico. En el enfoque microeconómico destacan una serie de desarrollos que intentan explicar cuáles son los factores que producen diferenciales en el ingreso entre las personas, así como en su productividad, y que ponen de relieve que el hecho de invertir en educación es una decisión que fácilmente puede ser equiparable a la decisión costo-beneficio que conlleva una inversión en capital. Importantes en este aspecto son los trabajos llevados a cabo por Mincer (1958), Becker (1965) y Schultz (1961).

Desde el punto de vista empírico, destacan una serie de trabajos que encuentran una relación positiva entre la educación y los efectos que esta tiene sobre los ingresos y la productividad. Algunos de los hallazgos de Becker (1975) son que la tasa de retorno de la educación es mayor para graduados universitarios hombres, de zonas urbanas, y menor para individuos con deserción escolar, mujeres y personas de zonas rurales. También observa que los graduados universitarios son más "dis- 
puestos" que los graduados del high school, y que la capacidad explica una parte relativamente menor de los diferenciales de salario que la educación universitaria. Heckman (1976) encuentra que el consumo, la oferta de trabajo y el perfil de ingresos de los individuos, se ven influidos por las diferencias en la capacidad de aprendizaje o dotaciones de capital humano.

Con respecto al enfoque macro, en la teoría sobre crecimiento económico se observa una amplia vertiente que imputa al capital humano las causas sobre el crecimiento de los países. En Solow (1956) se sientan importantes bases de lo que vendría al ser análisis de los factores que determinan el crecimiento, destacando los factores de capital y trabajo. Desde esa fecha, diferentes cuerpos teóricos han logrado incorporar el capital humano como un insumo más de producción (Lucas, 1988), y otros trabajos han hecho aportes empíricos sobre la relación entre el capital humano y el crecimiento del producto. Así se desprende del conocido Modelo de Solow "ampliado por capital humano" (Mankiew et al., 1992), junto con trabajos más recientes, que encuentran una relación positiva entre acumulación de capital humano, medida a través del número de años de escolaridad, y la tasa de crecimiento del producto per cápita (Bassanini y Scarpetta, 2001).

También es necesario reconocer que el aprendizaje se da a través de la práctica, tal como señala Arrow: "El aprendizaje sólo puede tener lugar a través del intento de resolver un problema y por lo tanto sólo tiene lugar durante la actividad" (1962: 155). Destaca ejemplos como el caso del "efecto Horndal", el que trata de una empresa de hierro que no tuvo inversiones significativas por un periodo de 15 años, y sin embargo, la productividad hora-hombre aumentó en un $2 \%$ cada año.

Desde una definición más amplia, destaca la visión de Sen (1997), que señala que los beneficios de la educación son mayores que las ganancias en rendimiento en la producción de bienes y servicios. La persona puede obtener otros beneficios de la educación por la "posibilidad de leer, argumentar, comunicar, elegir con mayor información, ser tenida en cuenta más seriamente por otros” (Sen, 1997: 70).

Junto con la literatura clásica, surgen en Chile una serie de trabajos que han relacionado la temática del capital humano al desarrollo del país y a la distribución del ingreso. Siguiendo a Brunner y Elacqua (2003), los países requieren del capital humano para desarrollarse, y así lo refleja la demanda que existe en el mercado laboral por personas con mayor destreza y nivel de conocimientos. 
Sin embargo, las condiciones de desigualdad de recursos, educacionales y culturales, en conjunto con la baja inversión, el bajo gasto por alumno, el bajo nivel de investigación relativo a otros países, entre otros, son factores que dificultan la formación de capital humano. Beyer (2005) destaca la interdependencia del capital humano y físico, y que su simple acumulación no basta, también se requiere de una serie de instituciones y políticas que aseguren la transformación de los recursos en capital productivo.

Entre los efectos no deseados de la desigual distribución de la educación se tiene la distribución del ingreso. Existe amplio consenso con respecto al efecto positivo que tendría la educación en la reducción de la brecha de los ingresos (Meller, 2000), sin embargo, también existe consenso con respecto a que es un factor de largo plazo, pues los resultados concretos de mejoras educacionales tardan en materializarse (De Gregorio 2006; Contreras 1999). De acuerdo a Contreras (1999: 330), la educación "explica alrededor de $40 \%$ de la desigualdad de los ingresos del trabajo y cerca de $80 \%$ de sus cambios".

El trabajo de Zamorano, González y Lagos (2010) es el primer intento por medir la productividad de los funcionarios municipales. El estudio efectúa una revisión de las principales teorías del capital humano municipal desde una perspectiva interdisciplinaria, y termina por medir la productividad de los funcionarios municipales, imputando como producto municipal el Índice de Desarrollo Humano Comunal 2003. Sin embargo, reconoce que la medida de producción municipal adoptada podría tener algún nivel de imperfección: "estos resultados requieren revisar el modelo diseńado en cuanto a la pertinencia de los indicadores de productividad del municipio y la búsqueda de opciones más representativas del quehacer y logros del municipio" (Zamorano et al., 2010: 45).

Centrando la discusión en el entorno de estudio, tal cual es el sector municipal, la literatura permite rescatar algunos elementos de interés para el tratamiento del problema. La productividad de las personas está intrínsecamente ligada al conocimiento, las habilidades y la experiencia que tienen. Sin embargo, tal como destaca la definición de Sen, este mayor nivel de capital humano trae aparejado otros efectos que también pueden incidir en los procesos de producción, tales como una mayor motivación de los funcionarios con respecto a su trabajo, una comunicación más efectiva, un mejor desempeño de los equipos, y en consecuencia, una mayor eficiencia en la gestión municipal. 


\subsection{Capital Humano y Sector Municipal}

El análisis del capital humano tradicionalmente se ha centrado en contextos donde el salario y la movilidad de las personas no se encuentran sujetos a restricciones considerables. Distinto es el caso de los funcionarios municipales, que se desenvuelven en un contexto donde los salarios, la movilidad, y otros aspectos contemplan rigideces, por lo que observar estas características es fundamental.

En SUBDERE (2005) se observa un interesante ejercicio que da luces con respecto a las características de los funcionarios municipales. El estudio consistió en un levantamiento de información en 104 municipalidades, que se tradujo en 1.060 encuestas a funcionarios, y entrevistas a directivos y encargados de personal entre otros. Los resultados señalan diferentes patrones entre los municipios rurales de bajos ingresos municipales y los urbanos de altos ingresos municipales.

Los municipios rurales de bajos ingresos tienden a tener un mayor nivel relativo de funcionarios menores de 30 años, y por ende una menor experiencia, menores salarios, un mayor porcentaje de trabajadores a contrata y a honorarios. También exhiben un menor porcentaje de profesionales y técnicos, y una mayor concentración de los funcionarios en las áreas de Desarrollo Comunitario y Dirección de Obras.

Los municipios urbanos de altos ingresos, que presentan un patrón totalmente distinto respecto a las características anteriores, tienden a tener una mayor concentración en áreas de Asesoría Jurídica, Control, y Administración y Finanzas. Esto es consistente con Valdebenito (2007: 13), que señala que a medida que disminuyen las carencias de una comuna, existe una menor demanda por servicios sociales, por lo que debería aumentar la demanda por otro tipo de bienes públicos. Siguiendo a SUBDERE (2005: 17) "Comunas con escasos recursos, deben concentrar sus esfuerzos cubriendo las necesidades básicas. Sólo cuando los recursos lo permiten, aumenta la presencia de funcionarios dedicados a las áreas administrativas y planificadoras".

En el análisis cualitativo, SUBDERE (2005) concluye que existen dos perfiles de funcionarios, uno peor preparado que presenta altos niveles de desmotivación y compromiso, versus otro que presenta las características contrarias. En el análisis de la política de recursos humanos destaca entre otros factores que generan des- 
motivación en los funcionarios, el hecho de que el reclutamiento, asignación de cargos y promoción responde más a factores políticos y de antigüedad.

Con respecto al liderazgo del alcalde y de los directores de unidad, el estudio señala que este es fundamental para el desempeño de la organización, y en las comunas urbanas de altos ingresos se observa una mayor carencia de estos liderazgos, debido a la cantidad de funcionarios, población comunal, y superficie (SUBDERE, 2005: 23). Caro (2005) encuentra según los parámetros de rendimiento municipal que define, que el buen desempeño se observa en municipios con alto nivel de fondo común municipal y con baja población, pero señala que esta información no permite establecer tendencias.

Dentro de las principales conclusiones de Zamorano et al. (2010) destaca el aumento significativo de los funcionarios municipales a inicios de la década del 2000, hecho que el estudio atribuye a la mayor complejidad de la gestión pública. También detecta un mayor promedio de funcionarios municipales profesionales en municipios de más de 100 mil habitantes, lo que concuerda con SUBDERE (2005), y un bajo nivel de profesionalización relativo al gobierno central. Sin embargo, destaca el que la productividad, en los términos definidos por su estudio, no es exclusiva de los funcionarios profesionales. De acuerdo a los autores, este hecho sugiere una coherencia con la teoría con respecto a que la productividad no estaría solo relacionada a la educación, sino que también se vería afectada por la experiencia, es decir, por el nivel de conocimiento y habilidad que los funcionarios han desarrollado como producto del desempeño de sus labores.

Avellaneda (2009), a través del análisis de 40 municipalidades colombianas, con datos para los años 2000-2004, encuentra que variables tales como la educación y la experiencia en los alcaldes influyen positivamente en la recolección de impuestos municipales, así como en el gasto en programas sociales. De la misma forma, Letelier (2010) encuentra que un mayor nivel de sueldo del alcalde, proxy de las habilidades de la plana mayor, implica una mayor probabilidad en la captación de fondos, con los beneficios que ello conlleva en la prestación de bienes públicos locales. 
Moon (2002) por otra parte, al explicar las razones que existen detrás de la adopción de prácticas de gobierno electrónico local, distingue entre dos tipos de administraciones, señalando que aquellas que son dirigidas por un administrador profesional son más propensas a adoptar innovaciones tecnológicas.

Lo anterior permite aseverar que un aumento de los niveles de capital humano municipal, entendiendo por esto un aumento en los niveles de educación, habilidades y experiencia, así como de todos los aspectos intangibles que afectan los anteriores factores, generaría una serie de beneficios en la gestión municipal.

Entre estos beneficios se tiene el mayor nivel de bienestar local, producto de la mayor absorción de las demandas locales que podría esperarse de funcionarios más comprometidos y capacitados para su labor. Esto constituiría una mayor eficiencia de los efectos que tiene el estado actual de la descentralización, en el contexto del teorema de la descentralización de Oates ${ }^{1}$. Es decir, dado el nivel de bienestar producto de la provisión de bienes públicos que realizan las municipalidades, un incremento de las capacidades y habilidades de los funcionarios para leer las necesidades de la comunidad local acarreará necesariamente un aumento del bienestar.

También, una mayor profesionalización podría incidir en cambios sustantivos en la gestión municipal, disminuyendo las sospechas teóricas de moral hazard en el comportamiento financiero de las municipalidades, y conformado evidencia empírica fundamental para el planteamiento de medidas reales de descentralización.

\section{UNA MEDIDA DE PRODUCCIÓN MUNICIPAL}

El concepto de productividad en el sector público siempre ha sido algo difuso debido a la complejidad que implica obtener una medida del producto de un organismo público. Stiglitz (2000: 231), analizando las diferencias organizativas entre el sector público y el privado, señala que el primero no actúa movido por beneficios, y se mueve más bien por objetivos políticos, que podríamos señalar tienen un carácter más amplio y social que la simple y objetiva rentabilidad.

Este teorema señala que existirá una mayor eficiencia toda vez que los niveles de provisión de bienes públicos locales encuentren una mayor correspondencia con la demanda local (ver Oates (1972)). 
En este contexto, la municipalidad es un organismo complejo desde diferentes dimensiones. Presta una serie de servicios en virtud de las diferentes áreas que la componen. En Valdebenito (2007), donde se realiza la estimación de un índice de demanda de servicios municipales, se observa un claro ejemplo de la heterogeneidad de las prestaciones que realiza la municipalidad solo para los servicios considerados, los que corresponden a servicios sociales, servicios a la comunidad, urbanismo, tránsito. Asimismo, SUBDERE (2005) señala como una de las principales características de los funcionarios la multiplicidad de funciones que deben realizar.

Debido a esto es que el plantear una medida de producto municipal es un ejercicio que implica cierto nivel de dificultad, tanto por la definición misma, como por la gran cantidad de aspectos que se pueden omitir al considerar una variable proxy del producto.

Sin embargo, existen hasta el momento algunas iniciativas que han tratado de medir el producto municipal. En Caro (2005) se construye un índice de desempeño, que vendría a representar el producto municipal, y que se calcula sobre la base de diferentes indicadores. El primero dice relación con la razón de gasto interno sobre gasto en la comunidad, el segundo es una razón de deuda sobre ingresos propios, el tercero mide el costo de la tramitación en el otorgamiento de patentes, y otros dos, que miden la evolución del SIMCE, y el cumplimiento de metas en salud. Mientras los tres primeros deberían ser lo más bajos posibles, con el objeto de que la municipalidad gaste menos en administración interna y más en la comunidad, exista un menor nivel de deuda, y mayor eficiencia en la tramitación de patentes, los últimos deben ser más altos.

Otras iniciativas se enmarcan en la aplicación de la técnica Data Envelopment Analysis (DEA), para la cual es necesario definir productos municipales. Uno de los primeros esfuerzos en esta línea se encuentra en Letelier L. y Muñoz R. (1998), donde los autores definen como outputs o productos municipales el SIMCE por año, el número de atenciones en salud primaria, y el Gasto en Bienes y Servicios a la comunidad. En otro artículo que sigue la misma metodología (Horst, 2007), la autora define como productos de la municipalidad la superficie en $\mathrm{m}^{2}$ de áreas verdes con mantención, el total de superficie de obras nuevas con destino habitacional aprobadas e iniciadas durante el año, el número de encuestas CAS aplicada por primera vez o actualizadas durante el año, y las toneladas de residuos sólidos domiciliarios. 
De acuerdo a las directrices señaladas en Zamorano et al. (2010), se debe considerar una medida más certera del producto municipal, pero también que considere una mayor representación del quehacer y de los logros municipales. En virtud de esto, se ha optado por usar el índice estimado por Caro (2005), y que usaremos como un proxy del producto municipal. En adelante usaremos indistintamente la palabra desempeño o producto municipal.

\section{PlanteAmiento DE Hipótesis}

A continuación plantearemos algunas hipótesis con respecto al efecto que variables asociadas al capital humano municipal podrían tener sobre la medida de producto municipal que usamos en este trabajo.

Una hipótesis obligada dice relación con el efecto que tienen los funcionarios sobre el producto de la municipalidad. Este efecto, que se supone opera a través de la educación, las habilidades y la experiencia en la gestión municipal, debería recaer en funcionarios indistintamente de su calidad de profesionales o no profesionales. Una medida inicial debería ser cómo influye el nivel agregado de funcionarios por habitante sobre el desempeño municipal. Sin embargo, no deberíamos esperar que el desempeńo aumentara indefinidamente a medida que el número de funcionarios municipales por habitante se incrementa. En el extremo, un número abultado de funcionarios comenzaría a crear ineficiencias y un bajo control de la gestión, tal como ocurre en el caso de una alta dotación de personal (SUBDERE, 2005).

En el Gráfico 1 se presenta una curva hipotética de producción municipal $(y)$, la que a su vez es función de la dotación de personal $(L)$, corregida por el número de habitantes de la comuna en cuestión $(P)$. Esta última variable corresponde al número de funcionarios municipales per cápita ${ }^{2}(l=L / P)$. Como se observa, la relación entre la dotación de personal y la producción es directamente proporcional, y a medida que la dotación aumenta, el aumento marginal en la producción es cada vez menor.

2 Se considera el número de funcionarios per cápita, pues al variar la población $(P)$ de una comuna a otra, y mantenerse el número de funcionarios $(L)$ constante, podría variar la eficiencia y la efectividad que el personal tiene en los procesos de producción municipal. 


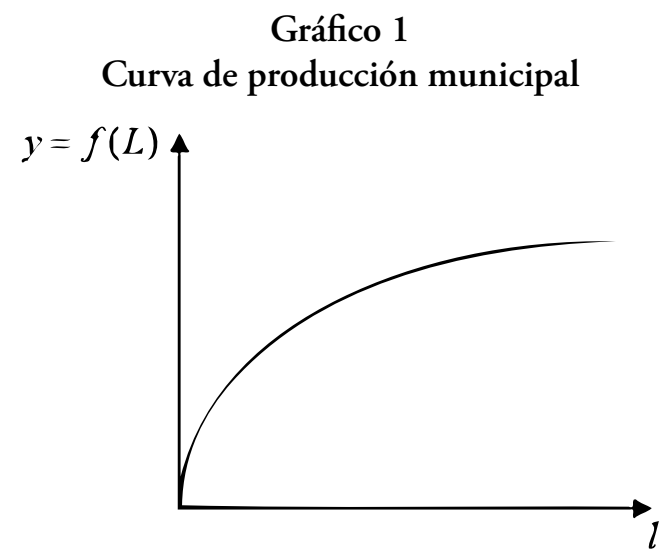

Formalmente, estas relaciones se observan a continuación:

$$
\frac{\partial y}{\partial l}>0 \quad \frac{\partial^{2} y}{\partial l^{2}}<0
$$

De acuerdo a lo anterior, es que se hace necesario testear los rendimientos decrecientes del número de funcionarios per cápita sobre el nivel de producto municipal, frente a lo que se espera un resultado que apoye la hipótesis de rendimientos marginales decrecientes.

No obstante, también se debe testear por el nivel de profesionalización, en virtud del aporte que los funcionarios profesionales efectúan sobre la gestión. El efecto del nivel de profesionalización se espera positivo sobre el producto, pues una mayor cantidad de profesionales podría incidir en los procesos de gestión, organización, y coordinación, entre otras cosas. Además, el bajo número de profesionales, que representan el 20-21\% de los funcionarios municipales (Zamorano et al., 2010), aleja la hipótesis de rendimientos marginales decrecientes para estos, y hace suponer un efecto considerable sobre el producto municipal.

Otro aspecto a tener en consideración en el producto municipal es el nivel de renta de los funcionarios, como un factor que podría afectar su desempeño. Letelier (2010), al explicar las causas del endeudamiento municipal, usa el grado del alcalde como un proxy de las habilidades del alcalde y el personal municipal, traduciéndose esta habilidad en una mejor captación de fondos. 
En este trabajo usaremos el grado del alcalde en la misma línea, como un proxy de las habilidades para la gestión municipal, pues la remuneración está relacionada al nivel de ingresos municipales (Miranda, 2011), y un mayor nivel de ingreso es sinónimo de una mayor complejidad organizacional, junto con una mayor profesionalización y especialización de los funcionarios (Gallego-Álvarez et al., 2010: 424). Debido a esto, se espera un efecto positivo del grado de salario del alcalde sobre el desempeño o producto municipal.

Una municipalidad que invierte más en sus funcionarios debería ser una municipalidad que tiene un interés importante por el capital humano, y por ende, por el perfeccionamiento y mejor desempeño de sus funcionarios. Este mejor desempeño en términos individuales debería traducirse en una sinergia que hace aumentar el desempeño organizacional. En vista de esto, se espera un efecto positivo del gasto en capacitación sobre el producto municipal.

De acuerdo a SUBDERE (2005) las comunas urbanas de altos ingresos tienen una mayoría relativa de funcionarios con mayor experiencia respecto a las rurales de bajos ingresos. Por el momento, ante la imposibilidad de contar con datos precisos sobre la experiencia de los funcionarios municipales, estimamos una medida que podría decir algo respecto de la experiencia agregada del personal. Esto es, si existen dos municipalidades con dotaciones de personal para los periodos $t_{1}, t_{2}$ $\mathrm{y}_{3}$, y la primera tiene una dotación $n$ para todos los periodos, mientras que la segunda tiene las dotaciones $n, m$ y $z$, para los tres periodos, donde $n<m<z$, se podría decir que la primera tiene un mayor nivel de experiencia agregada, debido a que los mismos funcionarios han adquirido experiencia para los tres periodos, mientras que para el caso de la segunda, se ha incorporado personal nuevo, sin la experiencia necesaria para la gestión municipal. Aun si la relación fuera $n>m$ $>z$, $\mathrm{o} n \neq m \neq z$, se tendría menos experiencia, debido a que se han ido o han entrado nuevos funcionarios. El efecto esperado de la variabilidad del personal, lo que debería acarrear una disminución de experiencia agregada en los equipos de trabajo, debería manifestarse en un efecto negativo sobre el producto municipal.

Una forma de capturar el nivel de variabilidad de los funcionarios, es la desviación estándar, que corresponde a un estadístico de dispersión. No obstante, esta medida no es capaz de capturar el efecto de la variabilidad de funcionarios sobre municipalidades de distintos tamaños, pues a medida que el número de funcio- 
narios es mayor, menos significativa será la variabilidad de los funcionarios, versus una municipalidad que tiene solo cinco funcionarios, donde la variabilidad tiene consecuencias importantes sobre el desempeño y el producto municipal. Para adecuar el estadístico a esta problemática es que usa el coeficiente de variación, que corresponde a la desviación estándar sobre la media. De esta forma, a medida que aumenta la media de funcionarios, disminuye el coeficiente de variación, quitándole importancia a la dispersión cuando el promedio de funcionarios es elevado 3 .

Entonces, se pretende usar la variabilidad de los funcionarios como un experimento que nos permita recrear un shock de rotación de personal. El resultado que esperamos de este shock sobre el producto municipal es negativo, pues un menor nivel de experiencia agregado implica un menor know how con respecto a la gestión municipal, y por ende, un menor logro de resultados.

Otra hipótesis interesante a investigar, es si los niveles de descentralización fiscal pueden influir sobre el producto municipal. Para esto usaremos una medida de descentralización fiscal estimada en Galilea et al. (2011:261). Esta medida corresponde a la proporción del ingreso municipal que queda disponible luego de hacer frente a los gastos en personal de planta. La lógica de este indicador es que el gasto en personal de planta corresponde a uno de los costos fijos del sector municipal, por lo que una mayor proporción que se dedica a conceptos que no tienen que ver con personal de planta, implican una mayor libertad en el uso del ingreso municipal, y por ende, un efecto positivo sobre el producto municipal.

No es posible ignorar en el desempeño o producto municipal, el efecto de variables que caracterizan a la demanda comunal, pues podrían influir en forma directa el perfil de la prestación de servicios que efectúa la municipalidad, su calidad y cantidad. Una aproximación se puede obtener a través de la urbanidad de la comuna.

3 La estimación de la desviación estándar muestral $\left(s_{L}\right)$ y el coeficiente de variación $\left(C V_{L}\right)$ se lleva a cabo a través de los siguientes estadísticos, donde $L$ corresponde al promedio de funcionarios para un periodo determinado:

$$
s_{L}=\sqrt{\frac{\sum_{i=1}^{n}\left(L_{i}-\bar{L}\right)^{2}}{n-1}} \quad C V_{L}=\frac{s_{L}}{\bar{L}}
$$


De acuerdo a los resultados del estudio de caracterización (SUBDERE, 2005), que indican un menor nivel de experiencia, profesionalización, y mayor rotación en el sector rural de bajos ingresos, es de esperar que el efecto de la urbanidad tenga un efecto positivo sobre el desempeño municipal.

También, dado que en sectores urbanos se observa una "mayor intensidad de uso de los espacios públicos y la mayor generación de residuos domiciliarios” (Irarrázaval, 2001: 11), se tiene en comunas urbanas un mayor monto de gastos en Servicios a la Comunidad, por lo que estas municipalidades gastarían más en la comunidad que en sí mismas. Recordando el sentido del primer indicador definido por Caro (2005), que las municipalidades deben gastar más en la comunidad que en la administración interna, estas comunas per se tendrían un mejor indicador de este tipo, por lo que el efecto de la urbanidad en el desempeño sería positivo.

Sin embargo, dado que en sectores urbanos, de mayor población, existe una pérdida de efectividad en el liderazgo del alcalde, también se esperaría un efecto negativo de la urbanidad en el desempeño. Serrano y Berner (2002) ofrecen evidencia con respecto a situaciones de indisciplina fiscal en el área de educación municipal debido a deficientes decisiones de política, las cuales se dan en comunas urbanas, populosas, de altos ingresos. De esto se podría inferir que en estas comunas existiría un mayor nivel de endeudamiento, considerando también la mayor demanda educacional relativa al sector rural. Todo esto se fundamenta en el hecho de que el índice de desempeño (Caro, 2005) considera una medida de endeudamiento municipal. La primacía de los efectos anteriores dependerá del signo del coeficiente para la variable población urbana.

Otra medida a considerar es el Índice de Desarrollo Humano, del cual se espera un efecto negativo sobre el producto municipal, debido a que comunas con un mayor índice representan comunas que han solucionado problemas básicos en materia de ingreso, salud y educación. Entonces, si el desempeño de la municipalidad converge a un estado de bienestar comunal específico, particular a cada comuna, aquellas comunas rurales de mayor vulnerabilidad son los espacios en los cuales más se puede avanzar en materia de resultados municipales.

Un aspecto a tener en consideración en el análisis de la productividad de los funcionarios municipales es la existencia de corporaciones, por lo que en estos casos no se podrían imputar los resultados de educación y salud sobre la productividad 
de los funcionarios de planta y contrata. Existe un número de 53 corporaciones para el caso de educación y 50 para salud (SINIM, noviembre de 2010). Sin embargo, de acuerdo al Sistema Nacional de Información Municipal 2009, de las 53 comunas que tienen corporaciones solo 4 no realizarían aportes municipales al área, mientras que de las 50 que tienen corporaciones de salud, 7 no realizarían aportes municipales al área (SINIM, noviembre de 2010).

De lo anterior se puede desprender que aunque los funcionarios municipales no administren directamente la salud y la educación para los casos en los que existen corporaciones, el aporte que realiza la municipalidad a las áreas de educación y salud es directamente proporcional al desempeño de los funcionarios de la municipalidad, esto pues una mayor eficiencia en la gestión que desarrollan impacta negativamente sobre los costos, y podría liberar recursos para aumentar el aporte que realiza la municipalidad a las áreas mencionadas.

\section{DESCRIPCIÓN DE LOS DATOS}

En esta sección se efectuará una revisión de los datos que usaremos en nuestro análisis. La unidad de observación de este estudio es la comuna, por lo que todas las variables que utilizaremos corresponden a datos de carácter municipal y comunal, y el universo corresponde a las 345 comunas del país. El análisis consiste en una estimación a través del método de mínimos cuadrados ordinarios (MCO) de una ecuación donde la variable a explicar es la producción municipal.

Como se observa en el cuadro 1, las variables que usaremos se dividen en variables de desempeño municipal, variables de capital humano, variables de descentralización fiscal y variables de demanda comunal. 


\section{Cuadro 1 \\ Definición de variables}

\begin{tabular}{|c|c|c|}
\hline Variable & Detalle & Fuente \\
\hline PRODUCTO_M & $\begin{array}{l}\text { Variable de desempeño municipal. Se calcula sobre } \\
\text { la base del inverso proporcional del indicador de } \\
\text { desempeño (ID). Esto es: } \\
\qquad \text { PRODUCTO_M } M=\frac{1}{I D}\end{array}$ & Caro, C. (2005). \\
\hline FUNCIONARIOS & $\begin{array}{l}\text { Porcentaje de funcionarios municipales de planta } \\
\text { y contrata sobre la población. }\end{array}$ & SINIM 2003. \\
\hline FUN_2 & Cuadrado de la variable FUNCIONARIOS & SINIM 2003. \\
\hline PROFESIONAL & $\begin{array}{l}\text { Razón entre funcionarios profesionales (planta y } \\
\text { contrata) sobre funcionarios municipales (planta } \\
\text { y contrata). Calculado sobre la base del indicador } \\
\text { disponible en SINIM, solo que no se encuentra en } \\
\text { porcentaje sino que en proporción. }\end{array}$ & SINIM 2003. \\
\hline GRADO & $\begin{array}{l}\text { Inversa del Grado de sueldos del Alcalde (GA). } \\
\text { Esto es: } \\
\qquad G R A D O=\frac{1}{G A}\end{array}$ & $\begin{array}{l}\text { SUBDERE, } 2010 . \\
\text { Formulación de } \\
\text { la Variable sobre } \\
\text { la Base de Letelier } \\
(2010) \text {. }\end{array}$ \\
\hline CAPACITA & $\begin{array}{l}\text { Porcentaje de gasto en cursos de capacitación } \\
\text { sobre gastos en personal. Indicador disponible en } \\
\text { SINIM. }\end{array}$ & SINIM 2003. \\
\hline COEF_VAR_FUN & $\begin{array}{l}\text { Coeficiente de variación del número de } \\
\text { funcionarios de la municipalidad para el periodo } \\
2001-2009 \text {. }\end{array}$ & SINIM 2001-2009. \\
\hline DFISCAL & $\begin{array}{l}\text { La medida de descentralización fiscal se estima } \\
\text { a partir de las variables ingreso total municipal } \\
\text { (ITM) y gasto en personal de planta (GPP): } \\
\qquad D F I S C A L: \frac{I T M-G P P}{I T M}\end{array}$ & $\begin{array}{l}\text { SINIM } 2003 . \\
\text { Formulación de la } \\
\text { Variable sobre la } \\
\text { Base de Galilea et al. } \\
(2011) .\end{array}$ \\
\hline IDH2003 & $\begin{array}{l}\text { Índice de Desarrollo Humano 2003. Elaborado } \\
\text { por el Programa de Naciones Unidas para el } \\
\text { Desarrollo junto al Ministerio de Planificación. }\end{array}$ & $\begin{array}{l}\text { PNUD y } \\
\text { MIDEPLAN } \\
\text { (2006). Las } \\
\text { Trayectorias del } \\
\text { Desarrollo Humano } \\
\text { en las Comunas de } \\
\text { Chile (1994-2003). } \\
\text { No 11, Temas de } \\
\text { Desarrollo Humano } \\
\text { Sustentable. }\end{array}$ \\
\hline POB_URBANA & Porcentaje de población urbana comunal. & SINIM 2003. \\
\hline
\end{tabular}


Dentro de variables de desempeño municipal se tiene el indicador estimado por Caro (2005) a partir de 5 indicadores de desempeño en diferentes áreas. El primer indicador rescata la situación ideal en la cual la municipalidad debería gastar más en la comunidad que en la administración interna, por lo que se define como la razón entre el gasto de administración interna sobre el gasto directo en la comunidad, y donde es deseable un valor más bajo de este indicador. El segundo indicador implica que la deuda municipal no debe sobrepasar los ingresos propios comprometiendo así el patrimonio municipal, lo que implica que la razón entre deuda e ingresos propios debe ser lo más baja posible. Un tercer indicador destaca el hecho de que las municipalidades deben ser promotoras del desarrollo económico local, para lo cual se usa un indicador que mide el costo y rapidez en el otorgamiento de patentes. El cuarto indicador considera la evolución del Simce para cuartos básicos durante el periodo 1999-2002. Por último, el quinto indicador considera metas determinadas entre los consultorios y el Ministerio de Salud.

A partir de estas variables, Caro (2005) construye un indicador de desempeño, que a menor valor implica un mejor desempeño o mayor producto municipal. Para efectos de este estudio, aplicando la inversa proporcional del indicador de desempeño, se obtiene una variable que denominamos PRODUCTO_M.

Las variables de capital humano han sido construidas en base a datos de SINIM del año 2003, pues el indicador de desempeño de Caro (2005) considera en su primacía, variables pertenecientes a dicho año. La primera variable corresponde al porcentaje de funcionarios municipales por habitante, variable que hemos denominado FUNCIONARIOS, y otra que corresponde a la misma variable pero elevada al cuadrado, esto es, FUN_2, con el objeto de explorar potenciales rendimientos decrecientes (ver Gráfico 1).

También se define la razón entre funcionarios de planta y contrata con título profesional sobre los funcionarios municipales, variable que definimos como PROFESIONAL, y que corresponde a la misma variable de profesionalización definida en SINIM pero expresada en proporción ${ }^{4}$.

4 La definición de las variables anteriores obedece al hecho de que comunas con un mayor número de funcionarios también tienen un mayor número de profesionales. Para aislar el efecto anterior en la estimación del modelo MCO, lo que produciría estimadores sesgados, la variable número de funcionarios se corrige por el número de habitantes, y la variable asociada a profesionales se corrige por el número de funcionarios municipales. 
Con el objeto de captar el efecto del salario del alcalde en el producto municipal, se usa el grado del alcalde, disponible en SINIM 2010. El grado más alto corresponde a " 1 ", mientras que el mínimo corresponde a " 6 ", por lo que se calcula la inversa proporcional del grado, obteniendo así la variable GRADO. Entonces, a medida que el grado aumenta de 6 a 5, la variable GRADO aumenta de 0.167 a 0.2 , reflejando el aumento en el salario.

Para testear por el nivel de capacitación, se obtiene de SINIM 2003, el gasto en capacitación como porcentaje del gasto en personal, y que se denomina CAPACITA.

Dada la imposibilidad de contar con cifras de experiencia del funcionario municipal, y de acuerdo a la discusión del punto 3, para medir la experiencia agregada o rotación del personal, se usa el coeficiente de variación del número de funcionarios para el periodo 2001-2009, por cada municipalidad. Cabe destacar que la medida es aplicada sobre los funcionarios municipales, de planta y contrata.

La variable de descentralización fiscal se obtiene a partir de SINIM 2003, usando las variables de ingreso total municipal y gasto en personal de planta. Así, se estima una ratio que indica la proporción de ingreso libre sobre el ingreso total, luego de financiar los gastos en personal de planta municipal.

Las variables de demanda comunal han sido construidas a partir de datos de SINIM y Chile Emprende. Dentro de estas tenemos el Índice de Desarrollo Humano 2003 (IDH2003), estimado por el Programa Naciones Unidas y el Ministerio de Planificación, y el porcentaje de población urbana presente en SINIM, que denominamos POB_URBANA.

\subsection{Estadística descriptiva de los datos}

En la Tabla 1 se puede ver la estadística descriptiva de las variables que usaremos. La variable desempeño, siguiendo a Caro (2005), tiene valor solo en cuanto permite ordenar el rendimiento de las municipalidades, y dado que en este trabajo ocupamos su inversa proporcional, su interpretación carece de un valor por sí misma.

De la tabla se desprende que en promedio, los funcionarios representan el $0.37 \%$ de la población de sus respectivas comunas, pues esta variable está expresada en 
porcentaje. La proporción de profesionales sobre funcionarios municipales alcanza en promedio la cifra de $0.217 \approx 0.22$, lo que indica que un quinto aproximadamente corresponde a profesionales. El promedio de la variable GRADO, 0.24, indica que la mayoría de los alcaldes tienen grados cercanos al límite inferior de salarios.

\section{Tabla 1}

Estadística descriptiva

\begin{tabular}{l|c|c|c|c|c}
\multicolumn{1}{c|}{ Variable } & Obs & Mean & Std. Dev. & Min & Max \\
\hline DESEMPENOO & 257 & 1.076 & 0.258 & 0.274 & 1.818 \\
\hline FUNCIONARIOS & 330 & 0.370 & 0.383 & 0.040 & 3.654 \\
\hline PROFESIONAL & 323 & 0.217 & 0.076 & 0.000 & 0.529 \\
\hline GRADO & 339 & 0.242 & 0.152 & 0.167 & 1.000 \\
\hline CAPACITA & 338 & 1.067 & 1.049 & 0.000 & 6.770 \\
\hline COEF_VAR_FUN & 261 & 0.135 & 0.142 & 0.015 & 0.753 \\
\hline DFISCAL & 338 & 0.851 & 0.039 & 0.708 & 0.944 \\
\hline POB_URBANA & 341 & 58.949 & 29.011 & 0.000 & 100 \\
\hline IDH2003 & 340 & 0.688 & 0.060 & 0.510 & 0.949 \\
\hline \hline
\end{tabular}

Fuente: elaboración propia a partir de base de datos construida.

Con respecto al gasto en capacitación, expresado como porcentaje del gasto en personal, este alcanza la cifra de $1.07 \%$. El coeficiente de variación para los funcionarios de contrata del periodo 2001-2009 alcanza en promedio la cifra de 0.135 , no obstante, su interpretación encuentra más sentido en el análisis propiamente tal, pues es una medida de relatividad en la variación de funcionarios de los diferentes municipios.

La variable DFISCAL implica que en promedio, a las municipalidades les queda un $85 \%$ de ingreso disponible para conceptos distintos del personal de planta. Por último, el índice de desarrollo humano y la población urbana tienen promedios de 0.688 y $58.95 \%$ de población urbana. 


\section{RESULTADOS DE LA ESTIMACIÓN}

Los resultados de la estimación a través del método MCO se observan en la Tabla 2 , en la cual se presentan los coeficientes estimados y los respectivos niveles de significancia.

La variable funcionarios per cápita tiene un coeficiente positivo y significativo en la mayoría de los modelos estimados, lo que es coherente con los resultados esperados, y refleja el aporte que los funcionarios municipales realizan sobre el producto municipal. Además, de acuerdo a la hipótesis de rendimientos marginales decrecientes, se tiene un coeficiente de signo negativo y significativo para la variable funcionarios per cápita al cuadrado $\left(F U N \_2\right)$, lo que indica que a medida que aumentan los funcionarios indefinidamente, llega un punto en el cual disminuye el producto municipal.

Con respecto al nivel de profesionalización se tiene un coeficiente positivo, de acuerdo a lo esperado, y significativo, en todos los modelos estimados.

Al controlar por la variable $G R A D O$, no se obtienen resultados significativos, por lo que no se puede rechazar la hipótesis nula de que este coeficiente es igual a cero, indicando la indiferencia de esta variable al explicar el producto municipal.

La variable de capacitación no presenta resultados importantes sobre el desempeño municipal, pues su coeficiente no es significativo, salvo el caso del modelo 3. Esto parece concordar con las conclusiones de SUBDERE (2005), en las cuales se señala que las capacitaciones no tienen coherencia y continuidad. Desde luego que es importante avanzar en la capacitación, pero debe ser un proceso sistemático y coherente con respecto a objetivos previamente definidos. Además su valor es ínfimo dentro del gasto en personal.

La variable de descentralización fiscal, DFISCAL, parece tener un efecto elevado sobre el desempeño en el modelo 5. Sin embargo, al controlar por variables asociadas a la demanda, pierde significancia (Modelo 6). Esto se produce por la alta correlación que existe entre variables asociadas a la demanda comunal y las mismas características municipales. 
Tabla 2

Regresión sobre desempeño municipal

\begin{tabular}{|c|c|c|c|c|c|c|c|}
\hline \multirow{2}{*}{$\begin{array}{c}\text { Variable } \\
\text { dependiente: }\end{array}$} & \multicolumn{7}{|c|}{ PRODUCTO_M } \\
\hline & (1) & (2) & (3) & $(4)$ & (5) & (6) & (7) \\
\hline Constante & $\begin{array}{c}0.828^{* * *} \\
(10.63)\end{array}$ & $\begin{array}{c}0.866^{* * *} \\
(9.93)\end{array}$ & $\begin{array}{c}0.853^{* * *} \\
(9.79)\end{array}$ & $\begin{array}{c}0.840^{* * *} \\
(8.92)\end{array}$ & $\begin{array}{c}0.0936 \\
(0.21)\end{array}$ & $\begin{array}{l}0.223 \\
(0.50)\end{array}$ & $\begin{array}{l}0.728 \\
(1.57)\end{array}$ \\
\hline FUNCIONARIOS & $\begin{array}{c}0.529^{* *} \\
(1.99)\end{array}$ & $\begin{array}{l}0.520^{*} \\
(1.93) \\
\end{array}$ & $\begin{array}{l}0.498^{*} \\
(1.86)\end{array}$ & $\begin{array}{l}0.542^{*} \\
(1.89) \\
\end{array}$ & $\begin{array}{c}0.609^{* *} \\
(2.11)\end{array}$ & $\begin{array}{l}0.543^{*} \\
(1.87) \\
\end{array}$ & $\begin{array}{l}0.398 \\
(1.38) \\
\end{array}$ \\
\hline FUN_2 & $\begin{array}{c}-0.494^{* *} \\
(-2.15)\end{array}$ & $\begin{array}{c}-0.485^{* *} \\
(-2.10)\end{array}$ & $\begin{array}{c}-0.481^{* *} \\
(-2.09)\end{array}$ & $\begin{array}{c}-0.514^{* *} \\
(-2.07)\end{array}$ & $\begin{array}{c}-0.557^{* *} \\
(-2.24)\end{array}$ & $\begin{array}{c}-0.544^{* *} \\
(-2.19)\end{array}$ & $\begin{array}{l}-0.299 \\
(-1.18) \\
\end{array}$ \\
\hline PROFESIONAL & $\begin{array}{c}0.728^{* * *} \\
(2.97)\end{array}$ & $\begin{array}{c}0.707^{* * *} \\
(2.90)\end{array}$ & $\begin{array}{c}0.628^{* *} \\
(2.55) \\
\end{array}$ & $\begin{array}{c}0.705^{* * *} \\
(2.62)\end{array}$ & $\begin{array}{c}0.625^{* *} \\
(2.30)\end{array}$ & $\begin{array}{l}0.542^{*} \\
(1.95)\end{array}$ & $\begin{array}{l}0.454^{*} \\
(1.67) \\
\end{array}$ \\
\hline GRADO & & $\begin{array}{l}-0.115 \\
(-1.15) \\
\end{array}$ & $\begin{array}{c}-0.0960 \\
(-0.95)\end{array}$ & $\begin{array}{l}-0.159 \\
(-1.35) \\
\end{array}$ & $\begin{array}{l}-0.135 \\
(-1.14) \\
\end{array}$ & $\begin{array}{c}-0.0450 \\
(-0.33)\end{array}$ & $\begin{array}{l}0.110 \\
(0.78)\end{array}$ \\
\hline CAPACITA & & & $\begin{array}{c}0.0289^{*} \\
(1.85)\end{array}$ & $\begin{array}{c}0.0232 \\
(1.35)\end{array}$ & $\begin{array}{c}0.0206 \\
(1.20)\end{array}$ & $\begin{array}{c}0.0225 \\
(1.31)\end{array}$ & $\begin{array}{c}0.0187 \\
(1.12)\end{array}$ \\
\hline CVAR_FUN & & & & $\begin{array}{r}0.0667 \\
(0.47) \\
\end{array}$ & $\begin{array}{c}0.0754 \\
(0.54) \\
\end{array}$ & $\begin{array}{l}0.0897 \\
(0.64)\end{array}$ & $\begin{array}{c}0.0492 \\
(0.36)\end{array}$ \\
\hline DFISCAL & & & & & $\begin{array}{c}0.876^{*} \\
(1.73) \\
\end{array}$ & $\begin{array}{l}0.821 \\
(1.62)\end{array}$ & $\begin{array}{c}1.266^{* *} \\
(2.48)\end{array}$ \\
\hline POB_URBANA & & & & & & $\begin{array}{c}-0.00117 \\
(-1.38)\end{array}$ & $\begin{array}{c}0.000438 \\
(0.45)\end{array}$ \\
\hline IDH2003 & & & & & & & $\begin{array}{c}-1.430^{* * *} \\
(-3.27)\end{array}$ \\
\hline $\mathrm{n}$ & 244 & 242 & 242 & 198 & 198 & 198 & 198 \\
\hline Prob $>$ F & 0.0027 & 0.0025 & 0.0013 & 0.0025 & 0.0016 & 0.0015 & 0.0000 \\
\hline Adj-R2 & 0.045 & 0.051 & 0.061 & 0.071 & 0.081 & 0.085 & 0.130 \\
\hline
\end{tabular}

$\mathrm{t}$ statistics in parentheses

${ }^{*} \mathrm{p}<0.1,{ }^{* *} \mathrm{p}<0.05,{ }^{* * *} \mathrm{p}<0.01$

En virtud de esto, el modelo más representativo corresponde al modelo 5, libre de los efectos de la demanda comunal, y que ejemplifica adecuadamente el efecto de los funcionarios sobre el producto municipal. Asimismo, permite aseverar que un mayor nivel de ingresos luego del gasto de planta, permite un mejor desempeño de la municipalidad (DFISCAL).

Sin embargo, pueden sacarse resultados interesantes de los modelos 6 y 7 . La incorporación de las dos variables de demanda comunal es en cierta medida re- 
dundante pues existe un grado de correlación entre estas (50\%), pero los efectos negativos que presentan sobre el producto, permiten apoyar las hipótesis planteadas en la sección anterior. Que las comunas convergen a un nivel de bienestar tal, del cual las urbanas se encuentran más cerca y las rurales más lejos, dada la cantidad de necesidades que aún se deben satisfacer en estas últimas. Y por otra parte, que en comunas urbanas de mayor complejidad existe una mayor dificultad en la gestión municipal, debido al mayor número de funcionarios y población comunal, entre otros aspectos (SUBDERE, 2005).

Como se desprende de la Tabla 3, si bien el indicador de gasto interno sobre gasto en la comunidad ofrece un mejor valor para las comunas urbanas de acuerdo a lo señalado por Irarrázaval (2001), pues estas municipalidades dadas las características comunales, gastan más en la comunidad, otros indicadores arrojan un mejor valor para comunas más rurales. Tal es el caso de la deuda municipal, la evolución en el Simce y el cumplimiento de metas en salud. Este hecho permite apoyar la hipótesis de que las comunas convergen a un nivel de bienestar del cual las rurales se encuentran más alejadas, y el hecho de que en sectores urbanos de mayor población existe una pérdida de efectividad en el liderazgo del alcalde y una mayor dificultad en la gestión.

Tabla 3

Promedios de los indicadores de desempeño por población urbana

\begin{tabular}{l|c|c}
\multicolumn{1}{c|}{ Indicador de desempeño } & Población Urbana $>50 \%$ & Población Urbana $<=50 \%$ \\
\hline Gasto Interno/Gasto Comunidad & .914 & 1.220 \\
\hline Deuda Municipal/Ingresos Propios & .206 & .047 \\
\hline Eficiencia en tramitación & 88.997 & 84.101 \\
\hline Evolución simce & -.006 & .005 \\
\hline Cumplimiento en salud & 1.273 & 1.336 \\
\hline \hline
\end{tabular}

Fuente: elaboración propia en base a Caro (2005). 


\section{CONCLUSIONES}

El estudio del capital humano municipal es relevante para países que aspiran a mayores grados de descentralización fiscal, entendiendo por esto un estado más cercano a las personas y que tiene la capacidad de absorber de forma efectiva las demandas de la ciudadanía.

El objetivo de este estudio fue determinar el aporte que hacen los funcionarios municipales sobre el desempeño municipal, avanzando en la precisión de los indicadores de producción municipal. De acuerdo a lo anterior, los principales resultados se articulan en torno a un efecto positivo de los funcionarios per cápita sobre el desempeño municipal, pero la existencia de rendimientos marginales decrecientes sobre el producto. Esto es, los funcionarios no pueden crecer en dotación hasta el infinito, pues esto produciría debilidades en el control de la gestión, y un debilitamiento en la intensidad del liderazgo del alcalde (SUBDERE, 2005), el que se reconoce fundamental en la gestión municipal.

Asimismo, la variable profesionalización es la más robusta para explicar la productividad de los funcionarios, lo que parece ser acorde con la teoría respecto al efecto que tiene la formación y la educación en el desempeño de las personas. No obstante, el nivel de profesionalización es bajo en términos generales, por lo que es importante avanzar en la profesionalización del sector. Esto sin duda, es un aspecto que gatilla en los resultados municipales, por lo que el estado debería incentivar la profesionalización del sector a través de incentivos concretos y programas de alta dirección municipal.

Si bien se encontró un modelo representativo (modelo 5), al incorporar condiciones asociadas al perfil de la demanda de las comunidades locales, ciertas variables pierden significancia, debido a la alta correlación entre las características municipales y las características comunales.

La profesionalización es importante para explicar la productividad de los funcionarios, sin embargo, los funcionarios en general también resultan relevantes en esta materia, en consistencia con los resultados obtenidos en Zamorano et al. (2010), que indican la no exclusividad de los profesionales en la productividad.

Con respecto a la capacitación, herramienta fundamental para aumentar el nivel de conocimiento y las habilidades de los funcionarios, los planes deberían orien- 
tarse a programas de mediano plazo, con metas bien definidas y coherentes con las necesidades de la municipalidad, y el tipo de funcionario que accede a estos programas.

Las restricciones financieras son fundamentales para explicar la mejor gestión municipal. Con respecto a esto, es fundamental avanzar en dos dimensiones, una que corresponde a una instancia de largo plazo, y que dice relación con aumentar el nivel de ingresos del cual las municipalidades disponen. Los países desarrollados tienden a tener un mayor nivel de gasto municipal sobre el gasto del sector público (Letelier, 2005), por lo que Chile debería avanzar, a medida que incrementa su nivel de desarrollo, en esa veta.

Sin embargo, no es posible avanzar en este camino si no existen mejoras en términos de eficiencia en la productividad municipal, y también en los aspectos que desincentivan y desmotivan a los funcionarios. De acuerdo a esto, es vital avanzar en el mayor uso de tecnologías, modelos de gestión e incorporar la innovación de los procesos municipales, sin dejar de lado el fortalecimiento de la carrera funcionaria y la profesionalización.

Sin duda, la mayor experiencia de los servidores públicos locales es fundamental en la gestión municipal, pues son las personas de más experiencia las que tienen un conocimiento acabado de los problemas, particularidades y consideraciones del trabajo local. En este sentido es vital avanzar hacia un banco de buenas prácticas que considere experiencias exitosas en materia de educación, salud, etc., usando para ello la tecnología de la que se dispone actualmente, la que ha disminuido los costos de la información de forma considerable.

Los resultados obtenidos son circunstanciales a los datos y las medidas de producción usadas, y si bien este trabajo nos ha llevado más allá, es menester seguir perfeccionando los indicadores de producción municipal, y la información de capital humano municipal.

Por último, como todo sistema complejo, las soluciones y mejoras surgen de diferentes actores, por lo que el abordar los problemas del sector municipal implica un dialogo conjunto, entre el Estado y las municipalidades, donde la riqueza en experiencia de estas últimas es un insumo fundamental para la mejor gestión municipal. 


\section{REFERENCIAS BIBLIOGRÁFICAS}

Arrow, Kenneth J. (1962). “The Economic Implications of Learning by Doing”. The Review of Economic Studies, Vol. 29, № 3, pp. 155-173.

Avellaneda, C. N. (2009). "Mayoral Quality and Local Public Finance". Public Administration Review, Vol. 69, $\mathrm{N}^{\circ} 3$, pp. 469-486.

Bassanini, Andrea y Scarpetta, Stefano (2001). "Does Human Capital Matter for Growth in OECD Countries?: Evidence from Pooled Mean-Group Estimates”. OECD Economics Department Working Papers 282.

Becker, Gary (1965). "A Theory of the Allocation of Time". The Economic Journal, Vol. 75, № 299. pp. 493-517.

Becker, Gary (1975). Human Capital: A Theoretical and Empirical Analysis, with Special Reference to Education. Chicago: National Bureau of Economic Research.

Beyer, Harald (1997). “Distribución del ingreso: Antecedentes para la discusión”. Estudios Públicos No 65, pp. 5-58.

Beyer, Harald (2005). "Productividad, Desigualdad y Capital Humano: Los complejos desafíos de Chile”. Estudios Públicos No 97, pp. 59-98.

Brunner, J.J. y Elacqua, G. (2003). Informe de Capital Humano en Chile. Santiago: Escuela de Gobierno, Universidad Adolfo Ibáńez.

Caro, C. (2005). Evaluación del Desempeño Municipal: ¿Cómo ordenar a los municipios según sus resultados? Santiago: Departamento de Estudios Municipales de la División de Políticas y Estudios, SUBDERE.

Herrera, Pedro y Francke, Pedro (2009). "Análisis de la eficiencia del gasto municipal y de sus determinantes". Revista Economía, Vol. 63, pp. 113-178.

Contreras, Dante (1999). "Distribución del ingreso en Chile. Nueve hechos y algunos mitos". Revista Perspectivas. Vol. 2, No 2, pp. 311-332.

De Gregorio, José (2006). "Economic Growth in Latin America: From the Disappointment of the twentieth Century to the Challenges of the Twenty-first". Central Bank of Chile, Working Papers $\mathrm{N}^{\circ} 377$.

Gallego-Álvarez, I.; Rodríguez-Domínguez, L. y García-Sánchez, I. (2010). “Are determining factors of municipal E-government common to a worldwide municipal view? An intra-country comparison”. Government Information Quarterly, Vol. 27, N 4. pp. 423-430. 
Heckman, James J, (1976). “A Life-Cycle Model of Earnings, Learning, and Consumption”. Journal of Political Economy, Vol. $84 \mathrm{~N}^{\circ}$ 4, pp. 11-44.

Horst, Bettina (2007). ¿Son nuestros municipios eficientes? Una aplicación del Modelo de Análisis Envolvente de Datos. Santiago: Instituto Libertad y Desarrollo.

Irarrázaval I. (2001). Financiamiento Municipal: Determinación de Funciones de Costo por tipo de provisión de servicios municipales y su financiamiento. Santiago: SUBDERE.

Letelier, L. y Muñoz R. (1998). Inversión Municipal y Eficiencia, Una aplicación del DEA en el caso municipal chileno. Paper presentado en la IFS International Conference in Córdoba.

Letelier, L. (2002). "Teoría y Práctica de la Descentralización. El caso de la Ley de Rentas Municipales II”. Estado, gobierno, gestión pública: Revista Chilena de Administración Pública, No 2, pp. 57-68.

Letelier, L. (2005). “Explaining Fiscal Decentralization”. Public Finance Review, Vol. 33 №2, pp. 155-183.

Letelier, L. (2010). "Theory and Evidence of Municipal Borrowing in Chile". Public Choice Vol 146, $\mathrm{N}^{\circ} 3-4$, pp. 395-411.

Lucas, R. E. (1988). "On the mechanics of economic development". Journal of Monetary Economics, Vol. 22, pp. 3-42.

Meller, Patricio (2000). Pobreza y distribución del ingreso en Chile (década del 90). Santiago: Centro de Economía Aplicada, Universidad de Chile.

Mincer, Jacob (1958). "Investment in Human Capital and Personal Income Distribution". The Journal of Political Economy, Vol. 66, N4, pp. 281-302.

Miranda, Víctor Hugo (2011). Jefe del Departamento de Finanzas Municipales, de la Subsecretaría de Desarrollo Regional y Administrativo, Ministerio del Interior. Entrevista.

Moon, M.J. (2002). "The Evolution of E-Government among Municipalities: Rhetoric or Reality?”. Public Administration Review, Vol. 62, N4, pp. 424-433.

Schultz, Theodore W. (1961). "Investment in Human Capital". The American Economic Review, Vol. 51, No1, pp. 1-17.

Sen, Amartya (1998). "Capital Humano y Capacidad Humana”. Cuadernos de Economía, Vol. XVII, N²9, pp. 67-72.

Sergio Galilea O., Leonardo Letelier S. y Katherine Ross S. (2011). "Descentralización de servicios esenciales. Los casos de Brasil, Chile, Colombia, Costa Rica y México en salud, educación, residuos, seguridad y fomento". Documentos de proyectos CEPAL N³71. 
Serrano, Claudia y Berner, Heidi (2002). "Chile: Un caso poco frecuente de indisciplina fiscal (bailout) y endeudamiento encubierto en la educación municipal”. RES Working Papers 3142.

Smith, A. (1776). "An Inquiry into the Nature and Causes of the Wealth of Nations". Versión en español en: Smith, A. (1958) Investigación sobre la naturaleza y causas de la riqueza de las naciones. México: Fondo de Cultura Económica.

Solow, Robert M. (1956). "A Contribution to the Theory of Economic Growth". The Quarterly Journal of Economics, Vol. 70, $\mathrm{N}^{\circ}$ 1, pp. 65-94.

Stiglitz, Joseph E. (2000). La Economía del Sector Público. España: Editorial Antoni Bosch.

SUBDERE (2005). "Investigación Caracterización del Funcionario Municipal”. Resumen Ejecutivo. Disponible en: http://www.subdere.gov.cl/1510/w3-article-66440.html

Valdebenito P., Jimena (2007). “Índice de Demanda de Servicios Municipales". Documento de trabajo. Santiago: Departamento de Políticas, SUBDERE, Ministerio del Interior.

Wallace E., Oates (1972): Fiscal Federalism, Nueva York, Harcourt Brace Jovanovich. Versión en español: Wallace E., Oates (1977). Federalismo fiscal. Madrid: Instituto de Estudios de Administración Local.

Zamorano, Leandro; González, Julián; Lagos, Roberto (2010). "Exploración al Capital Humano Municipal". Documento de trabajo, No 40. Instituto Chileno de Estudios Municipales, Universidad Autónoma de Chile. 